\title{
Determination of Geometrically Necessary Dislocations in Large Shear Strain Localization in Aluminum
}

Chaoyi Zhu*, Tyler Harrington*, Veronica Livescu', George T. Gray III', and Kenneth S. Vecchio*^

*Department of NanoEngineering, UC San Diego, La Jolla CA, 92131, 'Los Alamos National Laboratory, Materials Science and Technology Division, Los Alamos, NM 87545, ^^corresponding author, kvecchio@ucsd.edu

\begin{abstract}
In this paper, asystematic approach is presented to quantifying shear band evolution by quantifying geometrically necessary dislocations (GND) associated with morphological anisotropy in 7039aluminium alloy using the compact forced-simple shear (CFSS) design. A statistically motivated approach,i.e. the line averaged GND density profile, has been developed to investigate the GND density near heavily deformed, shear band regions. Our study shows that: i) line average GND density profiles for the Al samples machined in the A-direction (transverse to pancake-shaped grains), B-direction (parallel to longitudinal pancake-shaped grains, shearing in through-thickness direction), C-direction (parallel to pancake-shaped grains, shearing in the in-plane direction) and D-direction (parallel and through the pancake-shaped grains)are nominally similar; ii) apart from 7039-aluminum alloy C-direction that has a uniformGNDdistribution in the direction normal to shear due to a grain-sliding mechanism, GND profiles for other samplesdecrease steadily away from the shear band as plastic strain diminishes, in agreement with Ashby's theory of work hardening,iii) anisotropy in damage evolution and shearstress shear-strain response of 7039-aluminum alloy isassociated with the grain structure of the material, i.e. morphological anisotropy creating variations in grain boundary interactions; iv) microbands formation in D-direction is associated with local GND peaks; v) stress-relief crack propagating along grain boundaries due to the presence of voids or inclusions generates a 'shielding effect' on neighboring grains; and vi)the line average GND density profile within a single grain usually varies inversely with the width of the grain for A-,B- and D-directions, leading to generally pronounced higher GND density near triple junctions.
\end{abstract}


Nomenclature

$\begin{array}{cl}\mathbf{u} & \text { Displacement } \\ \boldsymbol{\beta}^{\boldsymbol{e l}} & \text { Elastic distortion } \\ \boldsymbol{\beta}^{\boldsymbol{p l}} & \text { Plastic distortion } \\ \varepsilon_{i j}^{e l} & \text { Element of the elastic strain tensor } \\ \omega_{i j} & \text { Element of the lattice rotation tensor } \\ \theta_{k} & \text { Element of the lattice rotation vector } \\ k_{i j} & \text { Element of the lattice curvature tensor } \\ \boldsymbol{\alpha} & \text { Nye dislocation tensor } \\ \delta_{i j} & \text { Element of the small deviation tensor } \\ \mathrm{g}_{i j} & \text { Element of the lattice orientation tensor } \\ \hat{\boldsymbol{l}} & \text { Normalized dislocation line direction } \\ \mathbf{r} & \text { Normal vector of an selected area } \\ \rho_{G N D} & \text { GND density } \\ \Delta \boldsymbol{\phi}_{d i s} & \text { Disorientation angle } \\ \boldsymbol{O}^{c r y} & \text { Crystal symmetry operators } \\ \boldsymbol{\Lambda} & \text { Lattice rotation gradient vector } \\ \boldsymbol{\xi} & \text { Active slip systems matrix } \\ \boldsymbol{\rho} & \text { Dislocation density vector }\end{array}$




\section{Introduction}

Localized unstable deformation of ductile metals and alloys when subjected to complex loading stress states and/or high-strain-rate-loading paths is most often associated with shear rupture (shear localization or shear bands). Shear localization and shear banding propensity has been extensively studied for more than half a century and several very detailed reviews of this work exist in the literature [1-36]. These studies have shown that shear localization depends on a number of aspects of the material microstructure and loading environment including: 1 ) physical properties of the material (heat capacity, thermal conductivity, thermal softening), 2) details of the mechanical loading to which the material is subjected (stress state, temperature, strain rate), and 3 ) details of the material microstructure including crystallographic texture, grain morphology and shape, dislocation density and distribution, and microstructural and phase stability $[3,4,6,15,18,36]$. Mechanics modelling, as well as numerous experimental studies of shear localization, has shown that a metal or alloy is prone to localized shearing during deformation if the material possesses a low strain/work-hardening rate and high degree of thermal softening under the loading conditions applied, a negative strain-rate sensitivity globally or locally within a material, and a low specific heat $[4,6,36]$. In addition, metals and alloys that undergo dynamic strain aging, strain-induced martensitic transformations, and crystalline to amorphous transitions under the loading rates and temperatures of interest are also more susceptible to shear localization [4, 6, 36]. The role of anisotropy (either crystallographically or morphologically-based within a material), in addition to directionality of loading relative to a materials microstructure, represent a current area of active shear-localization research aimed at the development of physically-based predictive modelling linking material processing to structure to properties to performance.

In spite of all this research, the study of shear localization still has significant limitations in terms of quantitative assessments of shear localized microstructure evolution. Nearly all studies to date have been unable to provide any numerical assessment of microstructures in shear bands, and initial microstructures that undergo shear localization have largely been treated as isotropic continuums. Recently, the introduction of the compact forced simple shear (CFSS) sample [37]enabled, for the first time, detailed quantitative examination of the influence of microstructural anisotropy on the evolution of shear localization and shear banding phenomena. Due to the simple design of the sample, the shear region can be oriented very accurately with respect to the microstructure, to probe grain anisotropy in either crystallographic texture or grain morphology texture.

With the advent of electron backscattered diffraction (EBSD), it is now possible to probe the evolution of deformation in terms of: initial grain textures, grain crystal rotations, and quantification of deformation in terms of geometrically necessary dislocations (GNDs) that are associated with lattice curvature. This latter aspect has been discussed in more general terms by Brewer et al., [38], Field et al., [39], and Calcagnotto et al., [40]. These studies demonstrate the ability and methodology to extract quantitative geometrically necessary dislocation (GND) densities from the lattice curvature as determined by EBSD within deformed samples. However, in each of these studies, the plastic deformation was limited to axial deformation and the extent of the deformation limited to less than $20 \%$ plastic strain.

The purpose of the present study is to combine the advantages of the CFSS sample for studying shear localization with respect to grain morphological anisotropy with EBSD analysis to extract quantitative differences in GND densities associated with shear localization in different microstructural orientations. Within the shear localizations regions, the plastic shear strain can easily exceed 1 , and because of the shear deformation mode, grain lattice curvatures approaching the shear band center can be very large. However, as will be demonstrated herein, with well-prepared samples for EBSD analysis, it is possible to obtain EBSD patterns from very close to the shear band centerlines, determine the lattice curvatures 
involved as a function of the microstructure orientation, and thus relate the extracted GND densities to differences in grain morphology. Lastly, by determining differences in the GND densities, it is possible to glean details related to local strengthening differences, clarify the operativedefect generation and storage mechanisms, and provide quantitative metrics for modeling of shear localization in ductile materials.

\section{Experimental}

\subsection{Materials}

In the present study, hot-rolled plate of 7039-Al that has a weak crystallographic through-thickness $<101>$ texture and a weak <001> rolling direction texture is analysed. 'Pancake-shaped' grains, highly elongated in the rolling direction, characterize the microstructure of the 7039-Al plate. The 7039-Al samples examined here are the same samples used in the previous study by Gray et al., [37]. Details of the materials, sample fabrication and orientation designations, sample testing procedures, and initial sample preparation for metallography can be found in this paper [37]. Additional polishing and etching methods were employed in this study to improve the quality of the EBSD data obtained from the shear band regions.

\subsection{Design of CFSS samples fromAl-7039 plate}

For analysis of the anisotropic dependence of simple shear mechanisms, the compact forced-simpleshear (CFSS) sample design depicted in Gray et al.,[37]is employed. The sample is designed in order to create a simple shear region parallel to the loading direction of the sample[37] in order to determine the effect of morphological anisotropy in Al-7039. Fig. 1 contains three images depicting the CFSS sample design from three viewing angles: perspective view, side vide and cross-section view.This CFSS sample design facilitates the alignment of specific microstructure feature to the shear direction, which is essential to the development of GND profiles with respect to the microstructure anisotropy. Machining of the specimen was done parallel or perpendicular to the 7039-Al plate's 'pancake-shaped' grains.

\subsection{Metallographic analysis}

For cross sectional analysis of the shear region, the samples were cut in half down the long axis perpendicular to the shear region. The cross sections were mounted in Struers Condufast ${ }^{\mathrm{TM}}$ mounting resin and polished to $0.05 \mu \mathrm{m}$ alumina. Prior to EBSD analysis, a final attack polish was performed on each sample to remove the mechanically deformed surface layer imparted during previous grinding and polishing.The attack polish solution for Al was $0.05 \mu \mathrm{m}$ alumina in a water suspension with $3 \% \mathrm{NaOH}$.

EBSD was conducted using a Bruker e-Flash EBSD detector on a FEI Quanta 600 SEM at 20kV and working distance around $15 \mathrm{~mm}$. To determine the angular resolution of the detector, a scan of a single crystal germanium sample was run using a step size of $200 \mathrm{~nm}$, and the misorientation distribution was plotted from $0-2^{\circ}$. The maximum number of occurrences of misorientation in this region was taken as the angular resolution of the system. In addition, step size analysis was carried out as detailed in the following section to ascertain the ideal number of pixels to be utilized for the GND calculation. To ensure that the center of shear band is aligned to the top edge of the EBSD scan, the shear-band crack was first aligned to the edge at the specified magnification of 500X and move horizontally to the region of interest. 


\section{GND Density Analysis}

\subsection{Theory}

To ascertain the geometrically necessary dislocation (GND) densities, the following theoretical approach is followed. Multiplicative decomposition of the deformation gradient [41] can be used to derive the additive decomposition of the displacement gradient tensor or distortion tensor $\boldsymbol{\beta}$ into plastic distortion and elastic distortion, while ignoring the higher order terms:

$$
F=F^{e} F^{p}=\left(I+\beta^{e l}\right)\left(I+\beta^{p l}\right) \approx I+\beta^{e l}+\beta^{p l}
$$

where $\boldsymbol{F}=\boldsymbol{I}+\boldsymbol{\beta}$ such that the distortion tensor is:

$$
\beta_{i j}=\partial_{j} u_{i}=\beta_{i j}^{e l}+\beta_{i j}^{p l}=\left(\varepsilon_{i j}^{e l}+\omega_{i j}\right)+\beta_{i j}^{p l}
$$

It is assumed that $\boldsymbol{\beta}$ for a continuum is a curl-free quantity. This is necessary to assure a single-valued continuous solution for the displacement field ' $\mathbf{u}$ ', where $\beta_{i j}=\partial_{j} u_{i}$. Since the distortion can be additively decomposed into plastic and elastic distortions, the curl of the plastic distortion is equal to the negative curl of the elastic distortion.

$$
\operatorname{curl} \beta=\operatorname{curl} \beta^{p l}+\operatorname{curl} \beta^{e l}=0
$$

Therefore, the Burger's circuit could be equivalently expressed in terms of both plastic and elastic distortion ${ }^{1}$. Using Stokes formula, the Nye tensor[42] could effectively be related to the elastic distortion.

$$
\begin{gathered}
\alpha=-\operatorname{curl} \beta^{p l}=\operatorname{curl} \beta^{e l} \\
B=-\oint \beta^{p l} d x=-\oiint \operatorname{curl} \beta^{p l} d S=\oiint \operatorname{curl} \beta^{e l} d S=\oiint \alpha d S
\end{gathered}
$$

Writing out the Nye tensor in indicial form yields the negative curl of lattice rotation and elastic strain, according to Kröner[43].

$$
\alpha_{i k}=-\epsilon_{k l j} \frac{\partial \beta_{i j}^{e l}}{\partial x_{l}}=-\epsilon_{k l j} \frac{\partial \omega_{i j}}{\partial x_{l}}-\epsilon_{k l j} \frac{\partial \varepsilon_{i j}^{e l}}{\partial x_{l}}
$$

Since the lattice rotation tensor is minus the curl of the lattice rotation vector, lattice curvature is related to the lattice rotation gradient as:

$$
\begin{gathered}
\omega_{i j}=-\epsilon_{i j k} \theta_{k} ; k_{i j}=\frac{\partial \theta_{i}}{\partial x_{j}} \\
\frac{\partial \omega_{i j}}{\partial x_{l}}=-\epsilon_{i j k} \frac{\partial \theta_{k}}{\partial x_{l}}=-\epsilon_{i j k} k_{k l}
\end{gathered}
$$

${ }^{1} \mathrm{~A} \mathrm{SF} / \mathrm{RH}$ convention was adopted to represent the Burger's circuit (Hirth and Lothe, 1982) 
Eq. (6)can be rewritten so that the Nye tensor is related to the lattice curvature tensor and elastic strain [43].

$$
\begin{aligned}
\alpha_{i k}=-\epsilon_{k l j} \frac{\partial \beta_{i j}^{e l}}{\partial x_{l}} & =-\epsilon_{k l j}\left(\frac{\partial \varepsilon_{i j}^{e l}}{\partial x_{l}}+\frac{\partial \omega_{i j}}{\partial x_{l}}\right)=-\epsilon_{k l j} \frac{\partial \varepsilon_{i j}^{e l}}{\partial x_{l}}+\epsilon_{k l j} \epsilon_{j k i} \frac{\partial \theta_{k}}{\partial x_{l}} \\
= & -\epsilon_{k l j} \frac{\partial \varepsilon_{i j}^{e l}}{\partial x_{l}}+\left(\delta_{k k} \delta_{l i}-\delta_{k i} \delta_{l k}\right) k_{k l}=k_{k i}-\delta_{k i} k_{m m}-\epsilon_{k l j} \frac{\partial \varepsilon_{i j}^{e l}}{\partial x_{l}}
\end{aligned}
$$

where the elastic strain is small compared with plastic strain, so the elastic strain is negligible. This is also known as the elastically rigid plastic approximation, where the plastic distortion of the lattice is assumed to be fully accommodated by the elastic lattice rotation alone[44]. However, there is a method available to extract the elastic strain, for example through cross-correlation of diffraction patterns method[45]. For this paper, the original Nye's relation is adopted, and the elastic strain is ignored[42]; this is thought to be appropriate given the very large strains under investigation in the sheared regions.

$$
\alpha_{i k}=k_{k i}-\delta_{k i} k_{m m}
$$

Explicit expression of the Nye tensor is written in Eq. (11). Lattice orientation gradients in the third direction (highlighted in red) are assumed to vanish[46, 47].Physically, it is sensible to argue that the gradient in the third direction is not zero. By setting the gradients to be zero, some dislocation types might be omitted. This assumption can also mean that the GND present must be summed up to have the gradients in the third direction to be zero, which is not necessarily true. Alternative methods involving only the experimentally determined terms have been previously presented by some authors [48-51], where five directly measurable components of Nye tensor: $\alpha_{12}, \alpha_{13}, \alpha_{21}, \alpha_{23}, \alpha_{33}$ and a difference term $\alpha_{11}-\alpha_{22}$ are considered. The $\alpha_{11}-\alpha_{22}$ term is valid only when elastic strain components are neglected [50]. Direct measurements of the lattice orientation gradients are also possible through FIB assisted serial-sectioning $[46,52]$ or 3D X-ray microscopy [44].

$$
\boldsymbol{\alpha}=\left[\begin{array}{ccc}
\frac{\partial \omega_{12}}{\partial x_{3}}-\frac{\partial \omega_{13}}{\partial x_{2}} & \frac{\partial \omega_{13}}{\partial x_{1}} & \frac{\partial \omega_{21}}{\partial x_{1}} \\
\frac{\partial \omega_{32}}{\partial x_{2}} & \frac{\partial \omega_{23}}{\partial x_{1}}-\frac{\partial \omega_{21}}{\partial x_{3}} & \frac{\partial \omega_{21}}{\partial x_{2}} \\
\frac{\partial \omega_{32}}{\partial x_{3}} & \frac{\partial \omega_{13}}{\partial x_{3}} & \frac{\partial \omega_{31}}{\partial x_{2}}-\frac{\partial \omega_{32}}{\partial x_{1}}
\end{array}\right]
$$

In order for the Nye relation to be applied in the EBSD technique, the lattice rotation tensor is approximated to the lattice orientation matrix for small deviations from the reference orientation[50].

$$
\begin{gathered}
\omega_{i j}=\mathrm{g}_{i j}-\delta_{i j} \approx \mathrm{g}_{i j} \\
\alpha_{i k}=-\epsilon_{k l j} \frac{\partial \beta_{i j}^{e l}}{\partial x_{l}} \approx-\epsilon_{k l j} \mathrm{~g}_{i j, l}
\end{gathered}
$$

It is by convention to choose the minimum misorientation or the disorientation angle from symmetryrelated solutions when only concerned about the misorientation angle [53], though physically solutions could also be selected based on the misorientation axis [54]. For cubic crystal such as Al, there are 24 
symmetry operators with maximum disorientation being $\sim 62.8^{\circ}[55]$. The equation used to find this disorientation angle is given in Eq. (14).

$$
\Delta \phi_{\text {dis }}=\min \left[\cos ^{-1}\left\{\operatorname{tr}\left[\left(O_{i}^{\text {cry }} \mathbf{g}_{A}\right) \mathbf{g}_{B}^{\boldsymbol{T}} O_{j}^{\text {cry }}\right]\right\}\right\}
$$

Here, $\mathbf{g}_{\mathbf{A}}$ is orientation matrix of the point of interest and $\mathbf{g}_{B}$ is the orientation matrix of the neighboring point. $O_{i}$ and $O_{j}$ are the symmetry operators, picked from the 24 groups of the cubic crystal. A threshold value of $2^{\circ}$ was used for the aluminium sample[56] to filter out GND density calculated based on minimum misorientation obtained from non-indexed points in the interior of the grain and points from the neighbouring grains/twins. If the minimum misorientation is found when $i=a$ and $j=b$ ( $a$ and $b$ are constants in the range of the number of symmetry operators), the lattice orientation difference is a second order transformation tensor, which can be calculated from the disorientation tensor $\Delta \mathbf{g}$, given by $\Delta \mathbf{g}=\mathbf{g}_{B} \mathbf{g}_{\boldsymbol{A}}^{-1}(\mathrm{i}=\mathrm{a}, \mathrm{j}=\mathrm{b})$ through Eq. (15):

$$
\mathbf{g}_{B}-\mathbf{g}_{\boldsymbol{A}}=(\Delta \mathbf{g}-\mathbf{I}) \mathbf{g}_{\boldsymbol{A}}
$$

The $g_{i j, l}$ is therefore the gradient of the lattice orientation difference [46], and $d_{1}$ is the step size in the direction of the lattice orientation gradient. In a square array EBSD scan, the $1^{\text {st }}$ neighbor would be the first layer wrapping the point of interest, forming a kernel. The kernel is the mask surrounding the EBSD point of interest as the central pixel in a $3 \times 3$ square of pixels. In this paper, we have defined the lattice orientation gradient to be between the point of interest to its $1^{\text {st }}$ neighbor, where the point of interest takes the mean orientation of that square kernel and serves as the reference orientation. The lattice orientation gradient could approximately be obtained by using Eq. (16), where the orientation gradient is assumed to be zero when $\mathrm{l}=3$.

$$
\mathrm{g}_{i j, l}=\frac{\mathrm{g}_{B_{i j}}-\mathrm{g}_{A_{i j}}}{d_{l}}
$$

On the other hand, Burger's vectors could be characterized using Frank's loop construction by relating the line vector $\hat{\boldsymbol{l}}$, slip direction $\boldsymbol{b}$ and the plane normal $\boldsymbol{r}$ :

$$
\boldsymbol{B}=\boldsymbol{b}(\hat{\boldsymbol{l}} \cdot \boldsymbol{r})=(\boldsymbol{b} \otimes \hat{\boldsymbol{l}}) \boldsymbol{r}
$$

Summing over individual dislocation densities yields the components of total dislocation density tensor, where each type of dislocation can be represented by a combination of $\hat{l}$ and $\boldsymbol{b}$ :

$$
\alpha_{i j}=\sum_{\mathrm{n}=1}^{N} \rho_{G N D}^{n} b_{i}^{n} \hat{l}_{j}^{n}
$$

As for FCC crystals, $(N=18)$, there are 18 dislocation configurations on $<110>\{111\}$ with6screwand12edge dislocations[46].In total, there are 18 types of geometric configurations of dislocations(36 if negative sign dislocations are considered) for aluminum. However, there are only nine equations that form a set of the underdetermined system with 36 unknowns. The six-term approximation involving terms $\alpha_{12}$, $\alpha_{13}, \alpha_{21}, \alpha_{23}, \alpha_{33}$ and $\alpha_{11}-\alpha_{22}$ for an underdetermined system are believed to have sufficient constraints since a minimum of five dislocation configurations are required for any arbitrary plastic deformation according to von Mises yield criterion. In this paper, a minimum condition was made about lattice orientation in the direction normal to the surface using the nine-term approximationand neglecting elastic strain, which is likely to result ina more accurate numerical estimation of the actual 
GND density content according to Ruggles et al.[49]. Methods of solving such a system of equations will be covered in the next section for Eq. (19).

$$
\alpha=\xi(9 \times 36) \cdot \rho(36 \times 1)=\Lambda(9 \times 1)
$$

\subsection{Minimization Schemes}

In order to solve for this underdetermined system\{Eq. 19\}, an appropriate minimization scheme was chosen to compute the 36 unknowns for 18 types of dislocation configurations in which the solution is folded back to give the original dislocation density vector. There are numerous combinations of dislocation configurations available that could be used to accommodate the same lattice orientation gradients of a crystal. The combination of a specific dislocation configuration with the lowest dislocation density or dislocation energy is believed to be what happens physically from a minimization principle standpoint. Three commonly employed minimization methods are: $L^{1}$-dislocation density minimization, $\mathrm{L}^{1}$-dislocation energy minimization, and $\mathrm{L}^{2}$-least squares of dislocation density minimization.

The fundamental assumption with the $L^{1}$ dislocation density minimization scheme is that it assumes edge and screw dislocations are equally likely to form in the material, such that the minimization scheme is established on the grounds of minimum total dislocation density. In fact, the elastic energy stored for edge dislocations is about $1 /(1-v)$ ( $v$ : Poisson's ratio) greater than for screw dislocations, therefore it is energetically less favorable to form edge dislocations than screw dislocations if the Burger's vectors of the two are the same. $L^{1}$ energy minimization scheme $\left(L^{1 e}\right)$ solves for a $\xi^{\prime}$ that minimise the total dislocation energy $\left(E_{G N D \text {, total }}^{L^{1 e}}\right)$ due to the presence of the GNDs, ignoring the interaction energy of the dislocations. Its governing equation is Eq. (20) where $\boldsymbol{E}_{\boldsymbol{N}}$ is the dislocation energy vector for the $\mathrm{N}$ types of dislocation configurations:

$$
\operatorname{minimum}\left\{E_{G N D, \text { total }}^{L^{1 e}}=\boldsymbol{E}_{\boldsymbol{N}} \cdot \boldsymbol{\rho} \mid \boldsymbol{\xi}^{\prime} \subset \boldsymbol{\xi}, \boldsymbol{\alpha}=\boldsymbol{\xi}^{\prime} \cdot \boldsymbol{\rho}\right\}
$$

$\rho_{G N D, \text { total }}^{L^{1 e}}=\mathbf{1}_{\boldsymbol{N}} \cdot \boldsymbol{\rho}_{\xi^{\prime}}$ is the total GND density, which corresponds to only a subset of $\xi$ that results in minimum total dislocation energy. For aluminium ( $b=2.86 \AA$ ), the Poisson's ratioaround 0.33 sets up the ratio of dislocation energy for edge and screw types nicely to be $1.5 \mathrm{E}_{\text {screw }}=\mathrm{E}_{\text {edge }}$.

To solve for the dislocation density vector $\rho$, an interior-point method using Matlab's 'linprog' command in the Optimization toolbox was utilized to solve for the medium scale minimization problem with a specified minimization scheme(http://www.mathworks.com/).

\subsection{GND Resolution Determination}

GND density calculations have limitations mainly deriving from the EBSD measurements. At large plastic strains, the lattice planes within the diffraction volume are not necessarily parallel, hence the Bragg angle for diffraction deviates slightly along the plane for diffraction. Consequently, blurring of the edges of the Kikuchi patterns, i.e. pattern degradation occurs, and indexing of the bands becomes almost impossible[57]. Therefore, GND density calculations on heavily deformed material could lack sufficient orientation data to compute the lattice orientation gradient.On the other hand, for materials with very low dislocation density, e.g. fully recrystallized $\mathrm{Cu}$, the calculated GND density risks false interpretation of the dislocation cell structures, which in reality is an arbitrary manifestation of the measurement noise. 
A simple analysis for an estimate of the lower bound of the GND density is presented below, where the EBSD based GND density resolution depends on several factors such as binning size, angular resolution and step size[58].

$$
\rho_{G N D}^{r e s}=\frac{\text { Angular Resolution }(\mathrm{rad})}{\text { Step size }(\mathrm{m}) \times \text { Burger' }^{\prime} \mathrm{s} \text { vector }(\mathrm{m})}
$$

In Eq. (21), the step size is calculated by dividing the actual map size by the number of pixels in both $x$ and $y$ directions for the 2D GND analysis.Asquare array of points for the EBSD was used so the step size in the $x$ and $y$ directionswould be the same. For our Bruker eFlash EBSD system on the FEI Quanta SEM 600 , the angular resolution was determined to be approximately $0.4^{\circ}$. Moreover, highly binned scans were found to display a higher angular resolution, i.e. higher GND noise floor,due to an increase in the read out noise added to the pixels[59]. Hence, the EBSD analysis was carried out at a binning size of $1 \times 1$ to minimise the effect of binning on angular resolution.

\subsection{Step Size Dependence Analysis}

An appropriate step size is a critical issue in analysing dislocation density since the step size must not be too large to overlook the variation in the dislocation cell structure, i.e. undulations in the lattice curvature, and not too small to compound EBSD orientation measurement error[60].A systematic approach of step size analysis is detailed here. It is suggested as part of the standard procedure prior to any EBSD based GND analysis.

In theory, the statistically stored dislocations (SSDs) generated via random trapping process were typically stored in diploes and multipoles. If the step size is small enough to separate the poles, SSDs will potentially be attributed as part of the GNDs. In most cases, decreasing the step size generally yields a greater fraction of GND density from the total dislocation density.Typically, the ideal step size is at the length scale of the dislocation cell structure or smaller in order to reveal and take into account most of the dislocation networks. However, smaller step size analysis is not only much more computationally expensive but also prone to measurement error. Determination of an appropriate step size therefore should be evaluated based on the dislocation cell structure, as well as, how much time and resources are available to spend on computation while maximizing accuracy of the measurements. Fig. 2 shows the results of a systematic analysis of the GND densities for the A-direction shear sample from the 7039-Al. The left portion of Fig. 2 contains a plot of the upper and lower limits of the calculated GND densities (indicated as the error bars) as a function of analysis step size, from 100nm to $1000 \mathrm{~nm}$. The mean value of the GND density is shown by the curve, and the calculated noise floor at each step size is shown as the open red circles. The plot reveals that at each step size the lower limit of the GND density is above the noise floor, indicating the measurement are valid relative to the measurement resolution. In the right portion of Fig. 2 are EBSD maps of the identical region in the shear sample of A-direction, away from the shear region, so relatively un-deformed. The images are taken at each step size from $100 \mathrm{~nm}$ to $1000 \mathrm{~nm}$, in $100 \mathrm{~nm}$ steps. The step size that best captures the dislocation cell structure within the grain is between $200 \mathrm{~nm}$ and $400 \mathrm{~nm}$. However, the data acquisition time for $200 \mathrm{~nm}$ step size is extremely long for the purpose of this study, and therefore a step size close to $300 \mathrm{~nm}$ was employed throughout. 


\subsection{Line-Averaged GND Density Profile (for simple shear experiment)}

As shown below in the EBSD scans in Fig. 3, the shear process is accompanied by a large extent of lattice rotation, the GND density associated with lattice rotation gradients would be expected to decrease away from the center of the shear band.

Single line profiles that are taken from a GND density map as a function of distance away from the center of the shear band (y) readily picks up specific local variation of GND and neglects the overall global variation with respect to morphological anisotropy. This suggests a more statistically robust approach, which essentially involves taking all the line profiles possible at every y position (normal to the direction of the shear band) and averaging them out in the $x$ direction (in the direction of shearing). In producing plots that present smooth data for interpretation, averaging for every $y$ value should be avoided, and instead plot at a specific interval, e.g. $12 \mu \mathrm{m}$ steps in the y direction for Fig. 6, Fig. 7, and Fig. 9. Additionally, the statistical reliability of the approach also dependents on how many available GND data points are used in the direction of shear for the generation of the line average GND profiles. Clearly, the more pixels available for each row of GND density averaging, the more reliable the result is, and as one approaches the shear band center, where EBSD data may be lacking, a greater uncertainty will result. In the following section, we will show that this technique can also be employed to quantitatively study microbands formation, examine the effect of grain shape on GND density, and assess the 'shielding effect' due to stress-relief cracking.

\section{Results and Discussion}

\subsection{Mechanical response of the materials}

The 7039-aluminumCFSS samples were preparedwith the shear direction aligned with various microstructuresincluding:A-direction (transverse to pancake-shaped grains), B-direction (parallel to longitudinal pancake-shaped grains, shearing in through-thickness direction), C-direction (parallel to pancake-shaped grains, shearing in the in-plane direction), and D direction (parallel and through the pancake-shaped grains). The damage evolution is found to be significantly different for the four directions, whereas load-displacement curves show similar mechanical behavior of them[37]. Moreover, load-displacement curves show that the B- and C-directions retain a higher level of shear strength at significantly larger shear strains, meaning an increased shear resistance in shearing parallel to the grains [37]. We will also show in the next section that the shear resistance can be reflected in the line average GND density profiles for these two directions. Additionally, results from Table 1 indicate that higher shear strength for the B- and C-directions are at the expense of overall ductility, particularly for the Cdirection. Reduced ductility insample $\mathrm{C}$ is thought to be due to the sliding of grains over each other along and parallel to the pancake-shaped grain boundaries.

In order to estimate the shear strain $(\bar{\gamma})$ of the CFSS samples, shear strain can be calculated by dividing the average shear displacement $(\overline{\mathrm{u}})$ by the shear band's width $(w)$. Using optical light microscopy, there are errors associated with making estimates of both the shear-band widths and average shear displacement, therefore an error ranging from $10 \%$ to $20 \%$ for the shear strain is expected.

As shown inTable 1, CFSS samples undergo severe plastic shear deformation, where the highest shear strain approaches 4 . Nevertheless, the following sections will show that we can, in practice, obtain reasonably good EBSD scans for GND analysis, except in the case of the C-direction aluminum alloy. The 
C-direction Al alloy sample exhibits poorly resolved EBSD images for clearly defining the shear-band width so it also has the highest error in shear strain estimate.

A through-thickness texture of 9.7 times random in $<101>$ is clearly shown for 7039-bulk aluminium alloy samples [37], and a characteristic pancake-shaped microstructure typical for rolled 7000 series Alalloys. Since rolled samples commonly have their texture anisotropically distributed, CFSS samples would therefore reflect the local texture of the locations where the samples were cut [e.g., A-direction (transverse to pancake grains) and D-direction (parallel and through the pancake grains)]. Previous EBSD studies have also shown that this local texture variation is not significant for the machined samples [37]. However, there still exists very different shear localization properties, see Fig. 3, for shear band formation and damage evolution mechanisms, which is believed to be related to the morphological anisotropy of the samples (i.e. anisotropy due to grain structure). For the EBSD scans, a comparison with EBSD scans in Gray et al. [37] confirms that EBSD scans presented in this paper coverthe region from the center of shear band to the slightly deformed region outside the heavily sheared regions.The damage evolution is clearly captured where the delamination and rotation of grains near the shear band occur. Upon closer examination, some cracking along grain boundary between inclusions or voidswere observed as well as void formation on the site of the inclusion.

Table 1. Estimate of shear strain $(\bar{\gamma})$ and error $\left(\boldsymbol{\sigma}_{\bar{\gamma}}\right)$ of the CFSS samples of 7039-aluminum alloy from average shear displacement $(\overline{\mathbf{u}})$ and shear-band width $(w)$.

\begin{tabular}{|l|l|c|c|c|c|}
\hline & & $\mathrm{w}(\mu \mathrm{m})$ & $\overline{\mathrm{u}}(\mu \mathrm{m})$ & $\bar{\gamma}$ & $\sigma_{\bar{\gamma}}$ \\
\hline \multirow{7}{*}{ 7039-Al } & $\mathrm{A}$ & 197 & 742 & 3.77 & 0.39 \\
\cline { 2 - 6 } & $\mathrm{B}$ & 190 & 482 & 2.54 & 0.28 \\
\cline { 2 - 6 } & $\mathrm{C}$ & 175 & 355 & 2.03 & 0.47 \\
\cline { 2 - 6 } & $\mathrm{D}$ & 201 & 793 & 3.95 & 0.40 \\
\hline
\end{tabular}

\subsection{Morphological anisotropy}

In addition, the variation oftexture of the machined samples is compounded by the lattice rotation associated with shear, making statistically valid GND measurements more complicated. Here, we utilize a line average GND density profile method covered previously in this paper to provide insight into the shear related lattice orientation gradients, see Section 3.5.

Fig. 3 shows EBSD images of the shear band regions of the A-, B-, C- and D-directionsamples, displayed in the first column to show the orientations of grains. In the second, third and forth columns, GND maps of total GND, edge-type GND and screw-type GND dislocations are plotted, respectively (log10 scale). Although screw dislocations are more energetically favorable to form than edge dislocations, the edgetype GND density is generally found to be, on average, much higher than screw-type GNDs as a result of more geometric configurations for edge dislocations. For each row of the figures, the sum of the GND map in the third and forth columns gives the total GND map in the second column for that specific direction. For the A-direction, transverse to the pancake-shaped grains, no local texture is present, and several cracks are shown along grain boundaries, which relieve stress due to severe shear localization. The 'shielding effect' due to crack formation will be presented in Fig. 4. For the B-direction, the shear direction is parallel to the longitudinal pancake-shaped grains in the through-thickness direction, which presents a mixed texture locally of $<001>$ rolling texture and $<101>$ through-thickness texture. The shear band region for the B-direction results in poorer pattern quality for EBSD compared with the Adirection, but the damage evolution of these two directions are quite similar due to similar grain 
morphology. Because of how grain shape varies along the direction normal to shear, it can be shown that GND density is inversely dependent on the grain thickness shown later in Fig. 6 . For the C-direction, the shear band is not clearly defined, and is aligned parallel to pancake-shaped grains in the in-plane direction, in which a very different shear mechanism is activated through grain boundary sliding, supported by the apparent pattern degradation of grain boundary regions. For the D-direction, the width of the shear band is very well defined, with the sample machined, parallel and through the pancake-shaped grains, but the shear region is comprised of very few grains. Microband formation is observed in this orientation, see Fig. 5, due to crystallographic slip and local shear across this small number of grains.

From the GND maps in Fig. 3, some GND 'hot' regions can be identified, where lattice rotation is obviously indicated by apparent colour changes due to the shear deformation. InFig. 3 A-direction, a large grain on the bottom left corner was found to have a low population of GND possibly due to a 'shielding' effect, as cracks propagating between inclusions or voids along its grain boundary. A separate GND study was carried out specifically using the line average GND density technique to reveal the variation GND density across two neighbouring grains, normal to shear direction, seeFig. 4, where a clear drop in GND density was captured to demonstrate the 'shielding effect' on the lower grain because of creation of free space due to cracking. The spacing created between grains effectively reduced the amount of GND necessary to satisfy compatibility requirements, whereas two triple junctions on the end of the grainaccumulated a largeramount of GND as a result of geometric constraint.

However, in some cases GND density data is insufficient to produce statistically reliable data at the center of shear-band, (see Fig. $3 \mathrm{D}$-direction, where the number of available GND density data points is lower than ten on the centerline of the shear band for D-direction sample). Due to the presence of a large fraction of grain boundaries, the poor EBSD pattern quality in the C-direction sample results in a poor GND density map, see Fig. 3. Nevertheless, the GND contents are in good agreement with lattice rotations apparent in the EBSD image.

Fig. 4 depicts how grain boundary cracks nucleated from voids or inclusions partially 'shield' the lower grain to severe shear localization. As shown in Fig. 4a, gaps or voids were created between grains during this deformation process, due to the amount of inclusions in the material. Total GND maps of two grains can be selected to create the image in Fig. 4b. This reduced the amount of GND present in the lower grain (Fig. 4b), which was otherwise necessary to ensure compatibility without the separation (i.e. cracking) between grains. The line average GND profile (Fig. 4c) clearly depicted the transition from a grain with high GND density (upper grain in Fig. 4b) to one with in general lower GND (lower grain in Fig. 4b). It is alsofound that the triple junctions between grains were hot spots for accumulation of GND. This figure effectively proves that grain boundary cracking is an effective means to reduce the amount of GND evolution.

Fig. 5a depicts an EBSD image of a grain (pale blue and yellow striped) that exhibited microband formation revealed in terms of orientation changes by slight colour variation within the grain (locations denoted by dotted lines). Fig. 5b shows the total GND density map in logarithmic scale created specifically for the isolated grain in Fig. 5 a containing the microbands. This data is used to quantitatively characterize the microband formation due to crystallographic slip in 7039-aluminum alloy using line average GND profile method given in Fig. $5 c$. When the line average GND profile approach is applied to this grain, a line plot is created that shows the part of the profile with variations of GND due to presence of microbands (Fig. 5c). Multiple local GND peaks associated with microbands are clearly shown along the shear direction at a distance of $200 \mu \mathrm{m}$ away from center of shear band of almost the same order of magnitudes. The accuracy of this approach to quantify the GND associated with microbands will be 
dependent on the resolution of EBSD technique and the method used to extract local lattice orientation gradients.

As shown in Fig. 5, there exists enough GND content to quantitatively reflect the distribution of GND associated with localized crystallographic microband formation. For the B-direction sample, the EBSD image in Fig. 3 displays a mixed texture composed of local texture of the sample in <101>and $<001>$ directions, but it has been shown to result in minor resistance to shear because only a slightly higher GND density value near shear band is calculated, see Fig. 9.

Fig. 6 shows EBSD and GND results for the shear band from the sample prepared in the B-direction. The results show that grain morphology does have an effect on GND density calculated using line average GND density technique. The intention of this plot is not to provide an exact relationship between slip distance and GND density, but to examine the trend between grain morphology (in a qualitative way) and GND. Fig. $6 a$ and $6 \mathrm{~b}$ show the combined EBSD and total GND maps of two selected grains (grain 1 in Fig. 6a and grain 2 in Fig. 6b). These two distinctly different grain shapes (grain 1 being very narrow and highly constrained, and grain 2 being a wider, less constrained structure) are analysed in terms of their GND profiles as a function of distance from the shear band center. The design of the CFSS sample allows the study of the effect of grain shape on total GND density across a wide range of shear deformation. Based on Ashby's work (1969), a lower slip distance or smaller grain thickness in this case, the greater the geometric constraint, and therefore a greater fraction of GND density needed to accommodate the constraint. The line average GND approach can be applied to these two grains and used to create the plotted data in Fig. $6 \mathrm{c}$; an averaging filter has been applied to smooth the data. The data reveal that for grains close to the maximum shear region, the GND densities are quite similar. However, as the shear strain decreases (i.e. moving down in Fig. $6(a, b)$, the separation between the red and black datasets increases, showing that the GND density differs between these two grains, which differ most distinctly by the grain thickness (i.e. level of constraint).

In Fig. 6, it is shown that the line average GND density changes inversely with respect to the slip distance, i.e. horizontal distance across the grain in the direction of shear, in accordance with Ashby's theory on the effect of slip distance [62]. In particular, grain 2 in Fig.6 displaysa gradual drop in GND density away from the shear band, indicating the diminishing effect of geometric constraintgeneration of GNDs. In comparison with grain 1, this grain shape has been found to betrivial at large shear strain regions since line average GND density profiles are of similar order of magnitude close the shear band whereas this effect is greatly enhanced at lower shear strain away from shear band, consequently leading to GND hot spots near triple junctions.

Fig. 7 demonstrates the effect of severe plastic deformation on pattern quality of EBSD scans as reflected by the number of successfully calculated GND data points. EBSD pattern quality can be undermined by severe plastic deformation, leading to low index rate. The number of GND data points is not the same as the indexed pixels in EBSD since several filters have been applied to produce the GND density maps, including the noise floor filter and the misorientation filter. The number of data points is counted in the shear direction and plotted with respect to distance away from shear band. The expectation is that GND data points will increase away from the shear band as a result of diminishing plastic shear strain. The number generally has been found to increase away from shear band. Since the line average GND density is statistical in nature, it is expected that this method is less reliable immediately adjacent to the shear band, where number of data points is limited (such as in the B- and D-directions). Clearly this data demonstrates the interpretation of such GND results very near shear band centers should be interpreted with care, dues to the limited number of usable EBSD data, however, 
in these regions, the shear strains have exceeded 1, and are much higher closer to 3-4. The results perhaps more clearly demonstrate that EBSD and GND calculations can be utilized in very highly strained samples.

From Fig. 3, the width of the shear bands among A-, B-, and D-sample shearing directions appear to be quite well defined at approximately $200 \mu \mathrm{m}$, whereas the shear band for the C-direction sample is poorly defined. The analysis of calculated GND density data points, see Fig. 7, reflects the quality of the EBSD scan as a function of distance radially from the center of the shear band, except for the C-direction sample, where the grains are horizontally aligned. A trend of increasing pattern quality away from shear band for the C-direction sample is observed, although it is not very well defined. This deterioration of the pattern quality [57]impliesthat the extent of plastic deformation for grains vertically aligned clearly increases at a closer distance to the center of the shear band.

Fig. 8 depicts compiled information regarding crystallographic orientation dependence of GND density as a function of Euler angle space. Since the GND density and orientation of a crystal after deformation varies greatly within one grain, the method adopted here is based on the grain averaged GND density calculated for each grain. These results are then plotted with respect to the mean orientation of that grain (post deformation orientation). It should be noted that grains being indexed depend on the misorientation angle set for determining a grain boundary. A single big fragmented grain might simply be post-processed as several small grains, but this should not affect this study significantly because of the averaging nature of this method. It is assumed also that the mean orientation of the grain is representative of the grain prior to deformation, since a great fracture of the curvature in bending of the grains is accommodated by a simple slip mechanism along the grain boundary rather than through lattice orientation gradients within the grain. It is found that for the A-direction, the grain average GND value ( $\log 10$ scale) is about 10.7 with a disperse distribution of points in the orientation space specified by Euler angles $\left(\varphi_{1}, \Phi, \varphi_{2}\right)$ due to lack of local texture. In the B-direction, the mean orientation of grains mostly have low angles in $\Phi$ and spans a wide range for $\varphi_{1}, \varphi_{2}$, but the grain average GND density (log10 scale) stays approximately the same for each grain at $\sim 10.5$. In the C-direction, a narrower span in the $\varphi_{1}$ and $\varphi_{2}$ angles, in the high angle range, and higher grain average GND density compared with other directions, consistent with results in Fig. 9. The results shown in D-direction are similar to Cdirection, but due to the small number of grains available, may not be representative. Overall, there is no specific correlation between the mean orientation (i.e. crystallographic texture) and GND evolution in these samples, and these results further highlight the influence of the grain morphology on the GND profiles in these 7039-Al sample shear bands.

In Fig. 9, line average GND profiles (discussed above) are plotted to study the effect of shear band formation on GND density evolution. The GND profiles for the A-, B- and D-direction samples decline steadily away from the center of the shear band, which agrees well with Ashby's theory on strain hardening due to the presence of GNDs. This trend is not observed for the C-direction sample where the shear band is poorly defined; instead a steady GND profile is exhibited. The line average GND profile of the four directions shown in Fig. 9 displays very similar order of magnitude for the total GND density and edge dislocations, but the screw dislocation density differs slightly. For samples shearing parallel to the grains, such as the B- and C-directions, the hardening effect at large strain is correlated with higher GND density near the shear band for the B-direction, and across the sample for C-direction.

The line average GND density profile of the four directions in Fig. 9 displays very similar order of magnitudes for the total GND density and edge dislocations, but the screw dislocation density differs slightly. For samples shearing parallel to the grains, such as the B- and C-directions, the hardening 
effectat large strain is correlated with higher GND density near the shear band for the B-direction and across the sample for $\mathrm{C}$-direction. Specifically for the C-direction, the line average GND density across the $y$-axis is in general higher than the other directions (i.e. higher shear resistance).From the average GND density within every grain plotted with respect to the mean orientation of the grain, nopreferential crystallographic orientation is observedin aluminum samples for generation of more GND, see Fig. 8. The GND density is in general more dependent on the microstructure of the aluminum samples, see Fig. 4, Fig. 5 and Fig. 6. For the C-direction, the grain average GND density is overall higher than other directions, consistent with the line average GND density profiles in Fig. 9. In conclusion, it is determined that the shear-induced local-lattice rotation for the A- and D-directions are not generally responsible for anisotropy in the deformation response of the 7039-aluminum alloy, whereas lattice rotation gradients for the B- and C-directions have been shown to enhance the shear resistance at the expense of ductility. Since the four directions possess little to no local bulk texture variation, the anisotropy observed must be mainly evolved due to the differences in the grain structure in the direction of simple shear (i.e. morphological anisotropy).

One additional point tonote is that the data points for the B- and D-direction samples at the far left should be interpreted with care because the average value of the GND density is calculated based on very few data points. There is uncertainty about how accurate these GND density data represent that particular region, though the order of magnitude suggests that these datalie in the reasonable range.

\section{Conclusions}

In this paper, a systematic method is presented, based on the emerging EBSD techniques, to quantitatively analyse the number density of geometrically necessary dislocations associated with simple shear of CFSS samples for metallic materials. These findings provide insights into the shear responses of morphologically and crystallographically anisotropic engineering materials. The presentfindings illustrate the effect of morphological anisotropy on damage evolution and shear band formation in 7039-aluminium alloys. The present observations are consistent with previouslypublished research on the shear response of materials, as well as the theories of dislocation generation and storage during plastic deformation. More specifically, it is observed that:

- The number of available GND density data points decreases nearer to the center of a shear band as a result of pattern degradation associated with the increasing plastic strain in the interior of a shear band.

- Away from the shear band, the line average GND density in general, for all types of dislocations, was noted to drop, consistent with Ashby's theory on work hardening of materials.

- While the deformation response and extent of lattice rotation was found to be similar for the Aand D-direction 7039-Al samples, the damage evolution differed including microbands/shear bands formation, void nucleation, crack propagation between inclusions along grain boundary each consistent withthe differences in the grain structure orientation relative to the shear direction,i.e. morphological anisotropy.

- Additionally, near all the shear bands, the nominal magnitudes of the GND total/edge dislocation densities were roughly the same for all the samples for the different grain structure/shear testing directions, whereas geometrically necessary screw dislocation densitiesgenerally differs from sample to sample.

- The increased shear resistance displayed by the B- and C-direction samples at large shear strains is thought to be due to a higher GND density near the shear band for the B-direction and an overall higher GND density across the C-direction sample. 


\section{Acknowledgements}

Los Alamos National Laboratory (LANL) is operated by Los Alamos National Security, LLC, for the National Nuclear Security Administration of the U.S. Department of Energy under contract DE-AC5206NA25396. The LANL work was partially sponsored by the Joint DoD/DOE Munitions Technology Development Program.Chaoyi Zhu would like to acknowledge insightful discussions with Dr. Ben Britton and Dr. Jun Jiang from Imperial College London.

\section{References}

[1] J.S. Rinehart, J. Pearson. Behavior of Metals Under Impulsive Loads. American Society for Metals, Cleveland, Ohio, 1954.

[2] H.C. Rogers. Adiabatic plastic deformation, Annual Review of Materials Science 9 (1979) 283-311.

[3] Y. Bai. Adiabatic shear banding, Res mechanica 31 (1990) 133-203.

[4] M.A. Meyers, Q. Xue, Y. Xu, V.F. Nesterenko. Microstructural evolution and self-organizationof shear bands. In: Chiba A, Tanimura S, Hokamoto K, (Eds.). Proc. 4th Int. Symp. on Impact Engineering. Amsterdam Elsevier, 2001. p.123-130.

[5] L.W. Meyer, F. Pursche. Experimental methods. in: B. Dodd, Y. Bai, (Eds.). Adiabatic Shear Localization: Frontiers and Advances. Elsevier, Amsterdam, The Netherlands, 2012. pp. 21-110.

[6] A.K. Zurek, M.A. Meyers. Microstructural aspects of dynamic failure. in: L. Davison, D. E. Grady, M. Shahinpoor, (Eds.). High-Pressure Shock Compression of Solids II. Springer, 1996. pp. 25-70.

[7] J.R. Klepaczko. Plastic Shearing at Very High Strain Rates, A Review. in: K. Kawata, J. Shioiri, (Eds.). Constitutive Relation in High/Very High Strain Rates. Springer-Verlag, Tokyo, 1996. pp. 59-68.

[8] N. Chung, J. Embury, J. Evensen, R. Hoagland, C. Sargent. Unstable shear failure in a 7075 aluminum alloy, Acta Metall Mater 25 (1977) 377-381.

[9] J.R. Klepaczko, H.V. Nguyen, W.K. Nowacki. Quasi-static and dynamic shearing of sheet metals, European Journal of Mechanics-A/Solids 18 (1999) 271-289.

[10] L. Anand, S.R. Kalidindi. The process of shear band formation in plane strain compression of fcc metals: effects of crystallographic texture, Mechanics of Materials 17 (1994) 223-243.

[11] A.S. Argon. Stability of plastic deformation. in: R. E. Reed-Hill, (Ed.). The In-homogeneity of Plastic Deformation. American Society for Metals, Metals Park, Ohio, 1973. pp. 161-190.

[12] L.W. Meyer, S. Manwaring. Critical adiabatic shear strength of low alloyed steel under compressive loading. International Conference on Metallurgical Applications of Shock-Wave and High-Strain-Rate Phenomena(EXPLOMET85), 1986. p.657-674.

[13] L.W. Meyer, E. Staskewitsch, A. Burblies. Adiabatic shear failure under biaxial dynamic compression/shear loading, Mechanics of Materials 17 (1994) 203-214.

[14] L.W. Meyer, L. Krueger, M. Gooch, M. Burkins. Analysis of shear band effects in titanium relative to high strain-rate laboratory/ballistic impact tests, Le Journal de Physique IV 7 (1997) 415-422.

[15] M. Meyers. Plasticity: Adiabatic shear localization. In: K. H. J. Buschow, R. W. Cahn, M. C. Flemings, B. Illschner, E. J. Kramer, S. Mahajan, (Eds.). Encyclopedia of Materials: Science and Technology: Elsevier, 2001. p.7093-7103.

[16] M.A. Meyers, Q. Xue, V.F. Nesterenko. Evolution in the patterning of adiabatic shear bands. In: M. D. Furnish, N. N. Thadhani, Horie Y, (Eds.). Shock Compression of Condensed Matter-2001. Melvile, New York: American Institute of Physics, 2002. p.567-570.

[17] T.W. Wright. Adiabatic shear bands. In: C. F. Chen, (Ed.). Proc. 11th US National Congress on Appplied Mechanics, vol. 43. New York: ASME, 1990. p.S196-S200. 
[18] T.W. Wright. Adiabatic shear bands: modeling and scaling laws. In: K. P. Staudhammer, L. E. Murr, M. A. Meyers, (Eds.). Fundamental Issues and Applications of Shock-wave and High-strain-rate Phenomena: Elsevier, 2001. p.457-466.

[19] Y. Xu, J. Zhang, Y. Bai, M.A. Meyers. Shear localization in dynamic deformation: microstructural evolution, Metallurgical and materials transactions A 39 (2008) 811-843.

[20] Q. Xue, V. F. Nesterenko, M.A. Meyers. Self-organization of shear bands in stainless steel: grain size effects. In: K. P. Staudhammer, L. E. Murr, Meyers MA, (Eds.). Fundamental Issues and Applications of Shock-wave and High-strain-rate Phenomena. New York: Elsevier, 2001. p.549-559.

[21] Q. Xue, M. Meyers, V. Nesterenko. Self-organization of shear bands in titanium and Ti-6Al-4V alloy, Acta materialia 50 (2002) 575-596.

[22] Q. Xue, X. Liao, Y. Zhu, G.T. Gray III. Formation mechanisms of nanostructures in stainless steel during high-strain-rate severe plastic deformation, Materials Science and Engineering: A 410 (2005) 252-256.

[23] Q. Xue, G.T. Gray III, B. Henrie, S. Maloy, S. Chen. Influence of shock prestraining on the formation of shear localization in 304 stainless steel, Metallurgical and Materials Transactions A 36 (2005) 1471-1486.

[24] Q. Xue, E.K. Cerreta, G.T. Gray III. Influence of Explosive-Driven Shock Prestraining on the Microstructural Evolution and Shear Localization of 304 and 316L Stainless Steels. In: M. D. Furnish, M. Elert, T. P. Russell, C. T. White, (Eds.). Shock Compression of Condensed Matter-2005, vol. 845. Melville, New York: American Institute of Physics, 2006. p.783-786.

[25] Q. Xue, G.T. Gray III. Development of adiabatic shear bands in annealed 316L stainless steel: Part I. Correlation between evolving microstructure and mechanical behavior, Metallurgical and Materials Transactions A 37 (2006) 2435-2446.

[26] Q. Xue, G.T. Gray III. Development of adiabatic shear bands in annealed 316L stainless steel: Part II. TEM studies of the evolution of microstructure during deformation localization, Metallurgical and Materials Transactions A 37 (2006) 2447-2458.

[27] Q. Xue, E.K. Cerreta, G.T. Gray III. Microstructural characteristics of post-shear localization in coldrolled 316L stainless steel, Acta materialia 55 (2007) 691-704.

[28] Q. Xue, J.F. Bingert, B.L. Henrie, G.T. Gray III. EBSD characterization of dynamic shear band regions in pre-shocked and as-received 304 stainless steels, Materials Science and Engineering: A 473 (2008) 279-289.

[29] Y. Zhang, G. Liu, X. Han. Analysis of strain localization for ductile materials with effect of void growth, International Journal of Mechanical Sciences 46 (2004) 1021-1034.

[30] K. S. Vecchio, U. Andrade, M. A. Meyers, L.W. Meyer. Microstructural evolution in high strain, high strain rate deformation. In: S. C. Schmidt, R. D. Dick, J. W. Forbes, D. G. Tasker, (Eds.). Shock Compression of Condensed Matter - 1991. Amsterdam: North-Holland, 1992. p.527-530.

[31] D. Rittel. The influence of temperature on dynamic failure mode transitions, Mechanics of Materials 30 (1998) 217-227.

[32] D. Rittel, Z. Wang, M. Merzer. Some experiments on adiabatic shear failure. Journal de Physique IV (Proceedings), vol. 134: EDP sciences, 2006. p.835-838.

[33] D. Rittel, Z. Wang. Thermo-mechanical aspects of adiabatic shear failure of AM50 and Ti6Al4V alloys, Mechanics of materials 40 (2008) 629-635.

[34] D. Rittel. A new perspective on adiabatic shear failure. DYMAT-International Conference on the Mechanical and Physical Behaviour of Materials under Dynamic Loading, vol. 2. Les Ulis, France: EDP Sciences, 2009. p.955-961.

[35] D. Rittel, S. Osovski. Dynamic failure by adiabatic shear banding, International Journal of Fracture 162 (2010) 177-185. 
[36] B. Dodd, Y. Bai. Adiabatic shear localization: frontiers and advances. in: B. Dodd, Y. Bai, (Eds.). Elsevier, Amsterdam, The Netherlands, 2012. pp. 1-12.

[37] G.T. Gray III, K.S. Vecchio, V. Livescu. Compact forced simple-shear sample for studying shear localization in materials, Acta Materialia 103 (2016) 12-22.

[38] L. N. Brewer, D.P. Field, C.C. Merriman. in: A. J. Schwartz et al, (Ed.). Electron Backscatter Diffraction in Materials Science. 2009. pp. 251-262.

[39] D.P. Field, C.C. Merriman, N. Allain-Bonasso, F. Wagner. Quantification of dislocation structure heterogeneity in deformed polycrystals by EBSD, Modelling and Simulation in Materials Science and Engineering 20 (2012) 024007.

[40] M. Calcagnotto, D. Ponge, E. Demir, D. Raabe. Orientation gradients and geometrically necessary dislocations in ultrafine grained dual-phase steels studied by 2D and 3D EBSD, Materials Science and Engineering: A 527 (2010) 2738-2746.

[41] E.H. Lee. Elastic-plastic deformation at finite strains, Journal of Applied Mechanics 36 (1969) 1-6.

[42] J.F. Nye. Some geometrical relations in dislocated crystals, Acta Metall Mater 1 (1953) 153-162.

[43] E. Kroner. Continuum Theory of Dislocations and Self-Stresses, Springer, 1958.

[44] B.C. Larson, A. El-Azab, W. Yang, J.Z. Tischler, W. Liu, G.E. Ice. Experimental characterization of the mesoscale dislocation density tensor, Philosophical Magazine 87 (2007) 1327-1347.

[45] A.J. Wilkinson, G. Meaden, D.J. Dingley. High-resolution elastic strain measurement from electron backscatter diffraction patterns: New levels of sensitivity, Ultramicroscopy 106 (2006) 307-313.

[46] E. Demir, D. Raabe, N. Zaafarani, S. Zaefferer. Investigation of the indentation size effect through the measurement of the geometrically necessary dislocations beneath small indents of different depths using EBSD tomography, Acta Mater 57 (2009) 559-569.

[47] S. Sun, B. Adams, W. King. Observations of lattice curvature near the interface of a deformed aluminium bicrystal, Philosophical Magazine A 80 (2000) 9-25.

[48] T.B. Britton, H. Liang, F.P.E. Dunne, A.J. Wilkinson. The effect of crystal orientation on the indentation response of commercially pure titanium: experiments and simulations, P R Soc A 466 (2010) 695-719.

[49] T. Ruggles, D. Fullwood. Estimations of bulk geometrically necessary dislocation density using high resolution EBSD, Ultramicroscopy 133 (2013) 8-15.

[50] W. Pantleon. Resolving the geometrically necessary dislocation content by conventional electron backscattering diffraction, Scripta Materialia 58 (2008) 994-997.

[51] J. Jiang, T.B. Britton, A.J. Wilkinson. The orientation and strain dependence of dislocation structure evolution in monotonically deformed polycrystalline copper, International Journal of Plasticity 69 (2015) 102-117.

[52] N. Zaafarani, D. Raabe, R.N. Singh, F. Roters, S. Zaefferer. Three-dimensional investigation of the texture and microstructure below a nanoindent in a $\mathrm{Cu}$ single crystal using 3D EBSD and crystal plasticity finite element simulations, Acta Materialia 54 (2006) 1863-1876.

[53] O. Engler, V. Randle. Introduction to texture analysis: macrotexture, microtexture, and orientation mapping, CRC press, 2009.

[54] W. Hutchinson, L. Ryde, P. Bate, B. Bacroix. On the description of misorientations and interpretation of recrystallisation textures, Scripta materialia 35 (1996) 579-582.

[55] A. Heinz, P. Neumann. Representation of orientation and disorientation data for cubic, hexagonal, tetragonal and orthorhombic crystals, Acta Crystallographica Section A: Foundations of Crystallography 47 (1991) 780-789.

[56] W. Cao, A. Godfrey, Q. Liu. EBSP investigation of microstructure and texture evolution during equal channel angular pressing of aluminium, Materials Science and Engineering: A 361 (2003) 914. 
[57] R. Keller, A. Roshko, R. Geiss, K. Bertness, T. Quinn. EBSD measurement of strains in GaAs due to oxidation of buried AlGaAs layers, Microelectronic Engineering 75 (2004) 96-102.

[58] A.J. Wilkinson, D. Randman. Determination of elastic strain fields and geometrically necessary dislocation distributions near nanoindents using electron back scatter diffraction, Philosophical Magazine 90 (2010) 1159-1177.

[59] J. Jiang, T.B. Britton, A.J. Wilkinson. Measurement of geometrically necessary dislocation density with high resolution electron backscatter diffraction: Effects of detector binning and step size, Ultramicroscopy 125 (2013) 1-9.

[60] B.L. Adams, J. Kacher. EBSD-based microscopy: Resolution of dislocation density, Computers, Materials, \& Continua 14 (2010) 185-196.

[61] F. Bachmann, R. Hielscher, H. Schaeben. Texture analysis with MTEX-free and open source software toolbox, Solid State Phenomena 160 (2010) 63-68.

[62] M. Ashby. The deformation of plastically non-homogeneous materials, Philosophical Magazine 21 (1970) 399-424.

Figure Captions:

Fig. 1. (left) Perspective view, (middle) side-view and (right) cross-section view of the CFSS sample.

Fig. 2. Left: Step size analysis for 7039-aluminum samples, machined from the A-direction. Black curve is the mean GND density of the selected area as a function of step size $(\mathrm{nm})$; Black error bars represent the range of GND density values spanned at each step size; red circles denote the noise floor estimated at every step size with a corresponding angular resolution of $0.4^{\circ}$ and Burgers vector of $2.86 \AA$ for aluminium; Right: GND maps in step size dependence analysis for the same selected area of 7039aluminum A-direction sample (size of the box is $25 \mu \mathrm{m}$ by $25 \mu \mathrm{m}$ ) at step sizes of $100 \mathrm{~nm}$, $200 \mathrm{~nm}, 300 \mathrm{~nm}, 400 \mathrm{~nm}, 500 \mathrm{~nm}, 600 \mathrm{~nm}, 700 \mathrm{~nm}, 800 \mathrm{~nm}, 900 \mathrm{~nm}$, and $1000 \mathrm{~nm}$. ( $\log 10$ scale, unit: $1 / \mathrm{cm} 2)$.

Fig. 3. 7039-Al alloy with shear zone aligned in the A-, B-, C-, and D-directions. Rows: 1st, A; 2nd, B; 3rd, C; 4th, D. Columns: 1st, EBSD image plotted with MTEX with selected example region showing the GND density map (Scale bar: 100 $\mu \mathrm{m}$ ) [61]; 2nd, total GND density map of the selected region ( $\log 10$ scale, unit: $1 / \mathrm{cm} 2$ ) with $20 \mu \mathrm{m}$ scale bar; $3 \mathrm{rd}$, GND density map of edge type dislocations (log10 scale, unit: $1 / \mathrm{cm} 2$ ) with $20 \mu \mathrm{m}$ scale bar; 4th, GND density map of screw type dislocations ( $\log 10$ scale, unit: $1 / \mathrm{cm} 2$ ) with $20 \mu \mathrm{m}$ scale bar.

Fig. 4. 7039-Al alloy with shear zone aligned in the A-direction. Two neighbouring grains selected to demonstrate the 'shielding effect' on the lower grain due to presence of voids or inclusions assisted stress-relief cracking along grain boundary using the line average GND density profile method. Red dotted line indicates the gain boundary, 
corresponding to a transition from a grain with high GND density to a one with lower

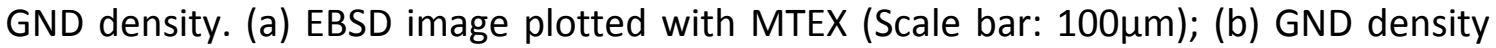
maps of two selected grains ( $\log 10$ scale, unit: $1 / \mathrm{cm} 2)$; (c) line average GND density profile across selected grains (unit: $1 / \mathrm{cm} 2$ ).

Fig. 5. 7039-Al alloy with shear zone aligned in the D-direction. One grain containing multiple microbands is selected and quantitatively analysed with the line average GND density profile method. Local GND density peaks corresponding to microbands are indicated

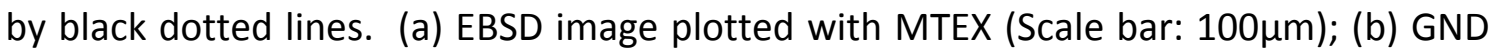
density maps of the grain with microbands (log10 scale, unit: 1/cm2); (c) line average GND density profile across microbands (unit: $1 / \mathrm{cm} 2$ ).

Fig. 6. 7039-Al alloy with shear zone aligned in the B-direction. Line average GND density profiles of grains of two different shapes: one of decreasing thickness of the grain away from the shear band and second one with increasing thickness. (a) EBSD image plotted with MTEX (Scale bar: $100 \mu \mathrm{m}$ ) and GND density map of grain 1 (log10 scale, unit: 1/cm2); (b) EBSD image plotted with MTEX (Scale bar: $100 \mu \mathrm{m}$ ) and GND density map of grain 2 (log10 scale, unit: 1/cm2); (c) line average GND density profile across the two grains (unit: $1 / \mathrm{cm} 2$ ).

Fig. 7. Number of available GND data points as a function of distance away from the center of shear band for line average GND density analysis in 7039-aluminum alloy machined in A-, B-, C- and D- directions.

Fig. 8. Effect of grain average orientation on grain average GND density (log10 scale, unit: $1 / \mathrm{cm} 2)$. Grain average GND plotted with respect to three Euler angles $\left(\boldsymbol{\varphi}_{1}, \boldsymbol{\Phi}, \boldsymbol{\varphi}_{2}\right)$ defining the mean orientation of the grains in CFSS 7039-aluminum alloy samples machined from A-, B-, C-, and D-directions.

Fig. 9. Comparison of line average GND density as a function of distance away from the center of shear band for (left) total GND density, (middle) edge type GND density and (right) screw type GND density between 7039-Al alloy samples machined in A-, B-, C- and Ddirections (log10 scale). 

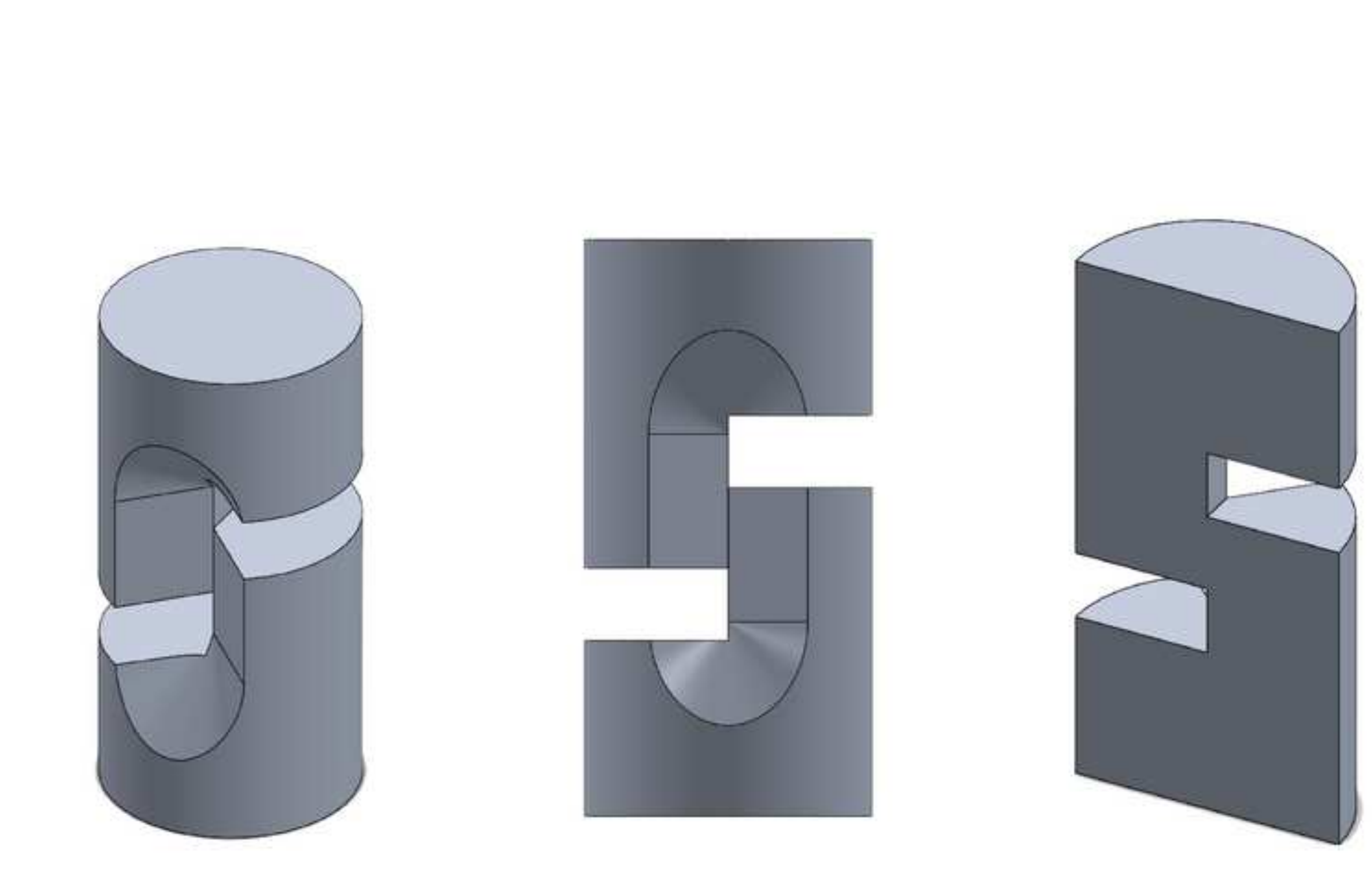

\author{
Figure
}

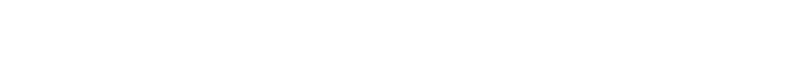

.

.



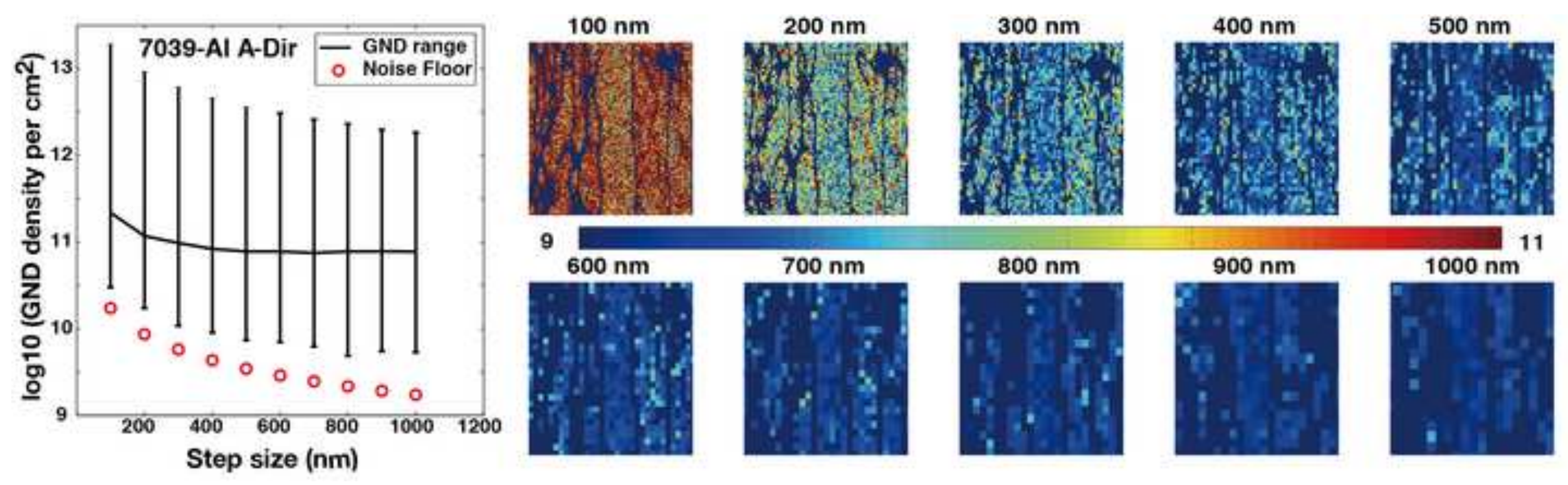

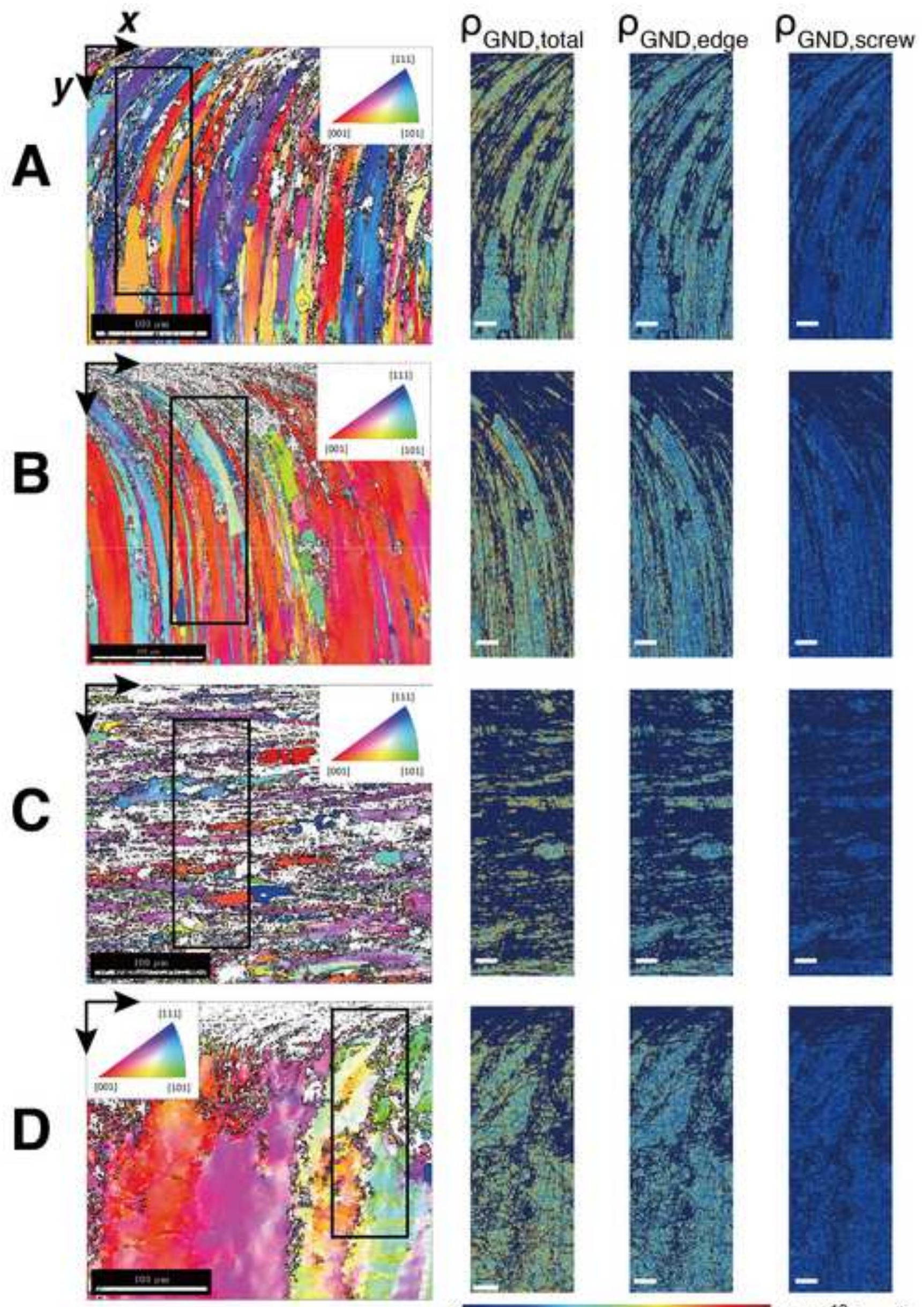

0

$10 \times 10^{10}\left[\mathrm{~cm}^{.2}\right]$ 


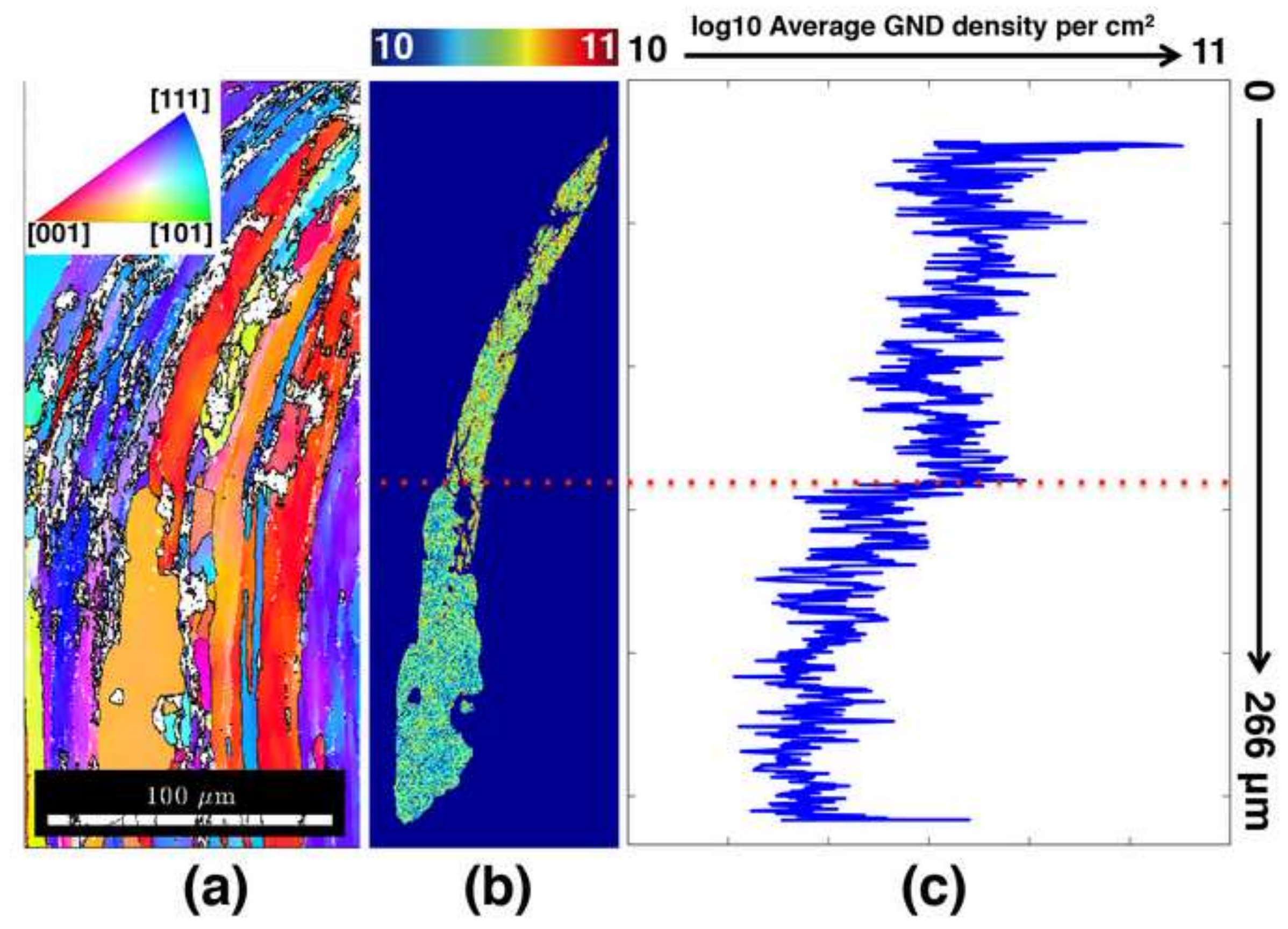

今

(b)

(c) 


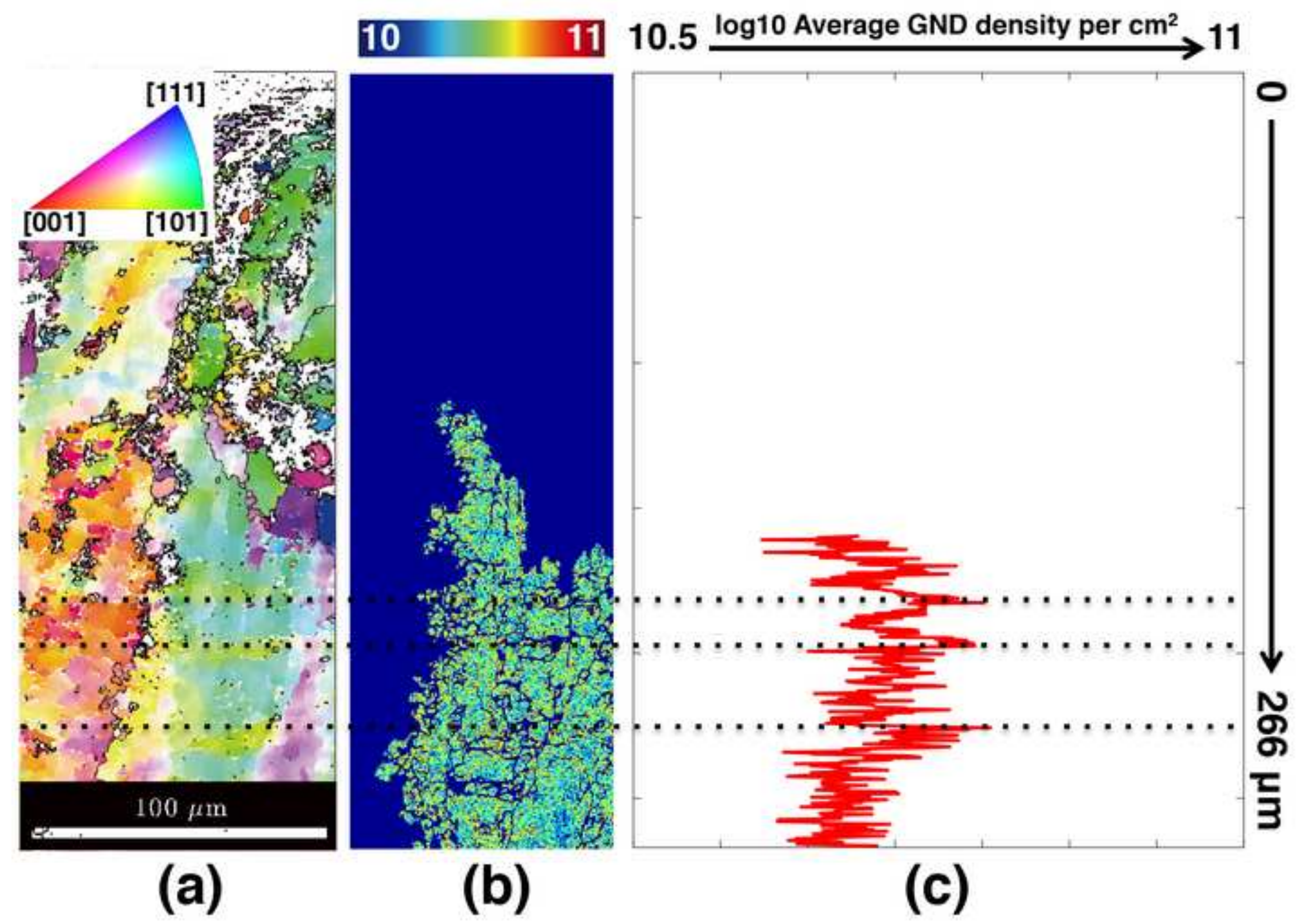

(a)

\section{0 $10.5 \longrightarrow 11$}

(b) (c) 


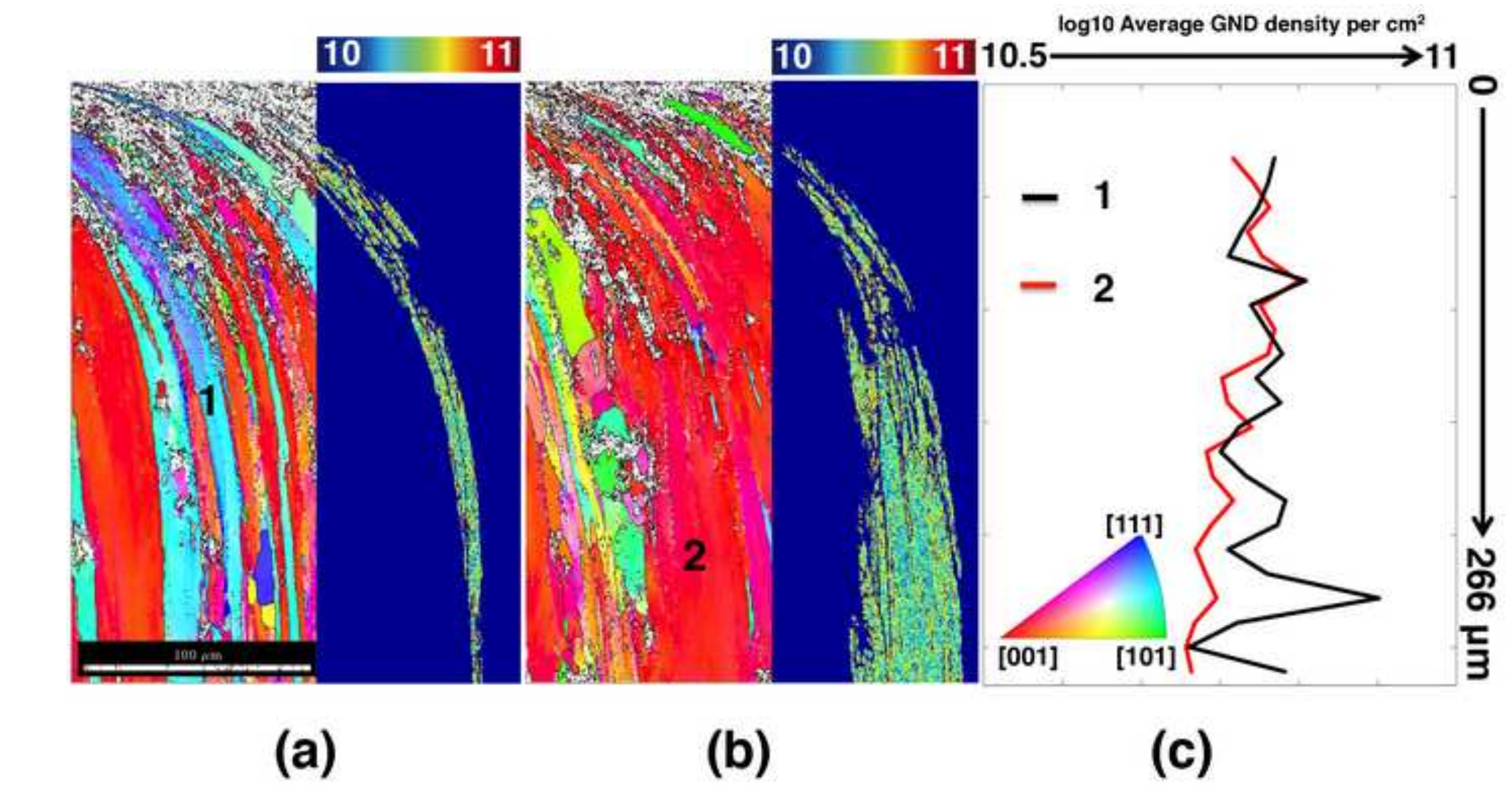

(a) (b) (c)

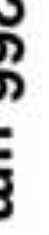$$
\text { . }
$$ 


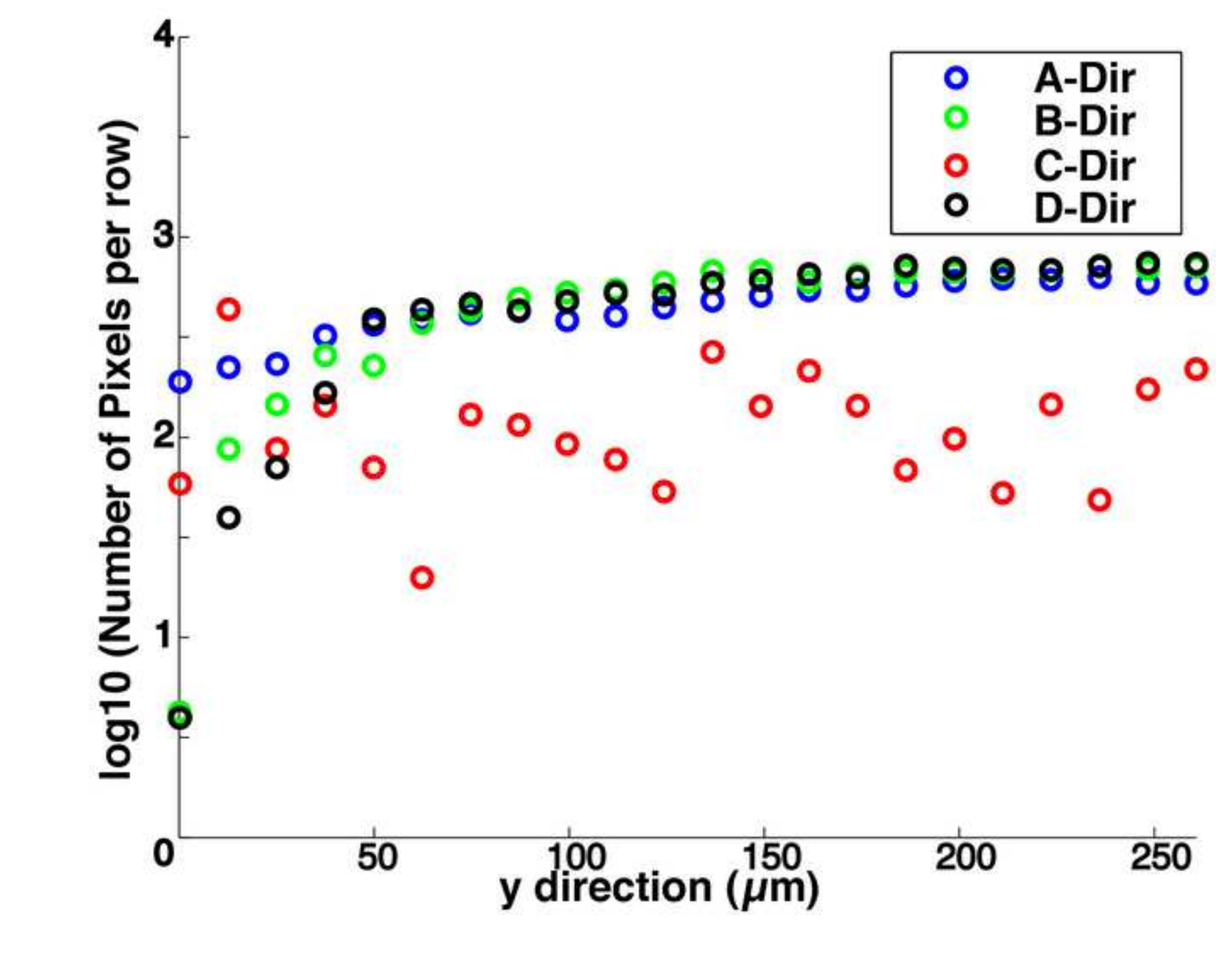



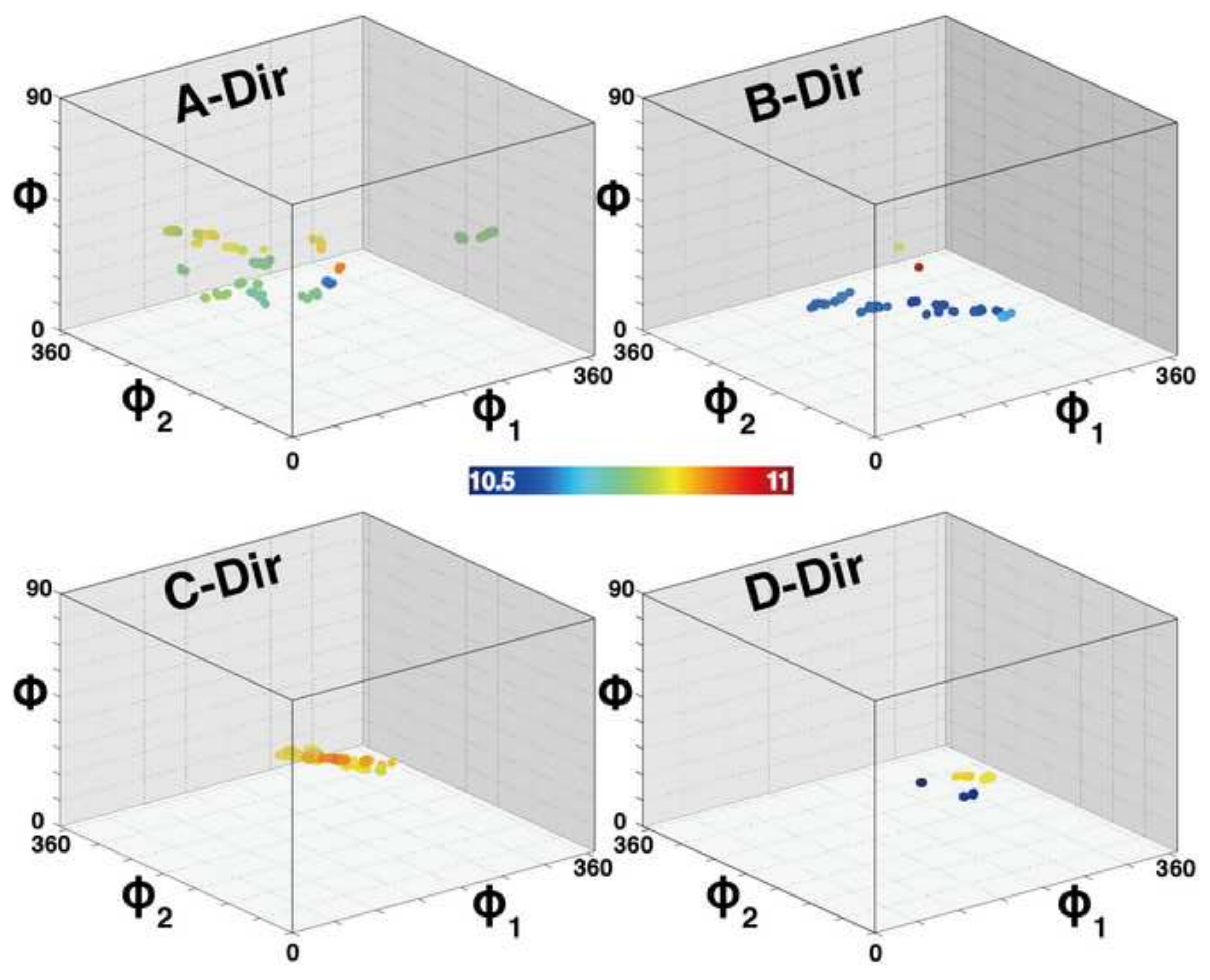

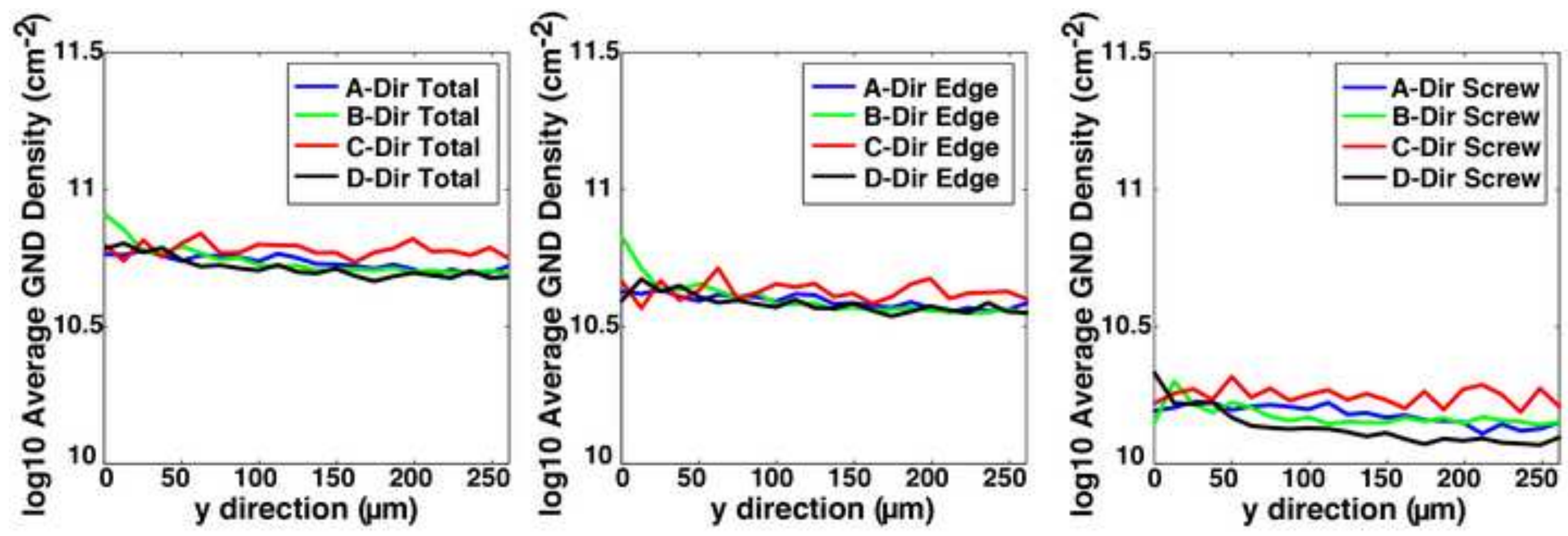


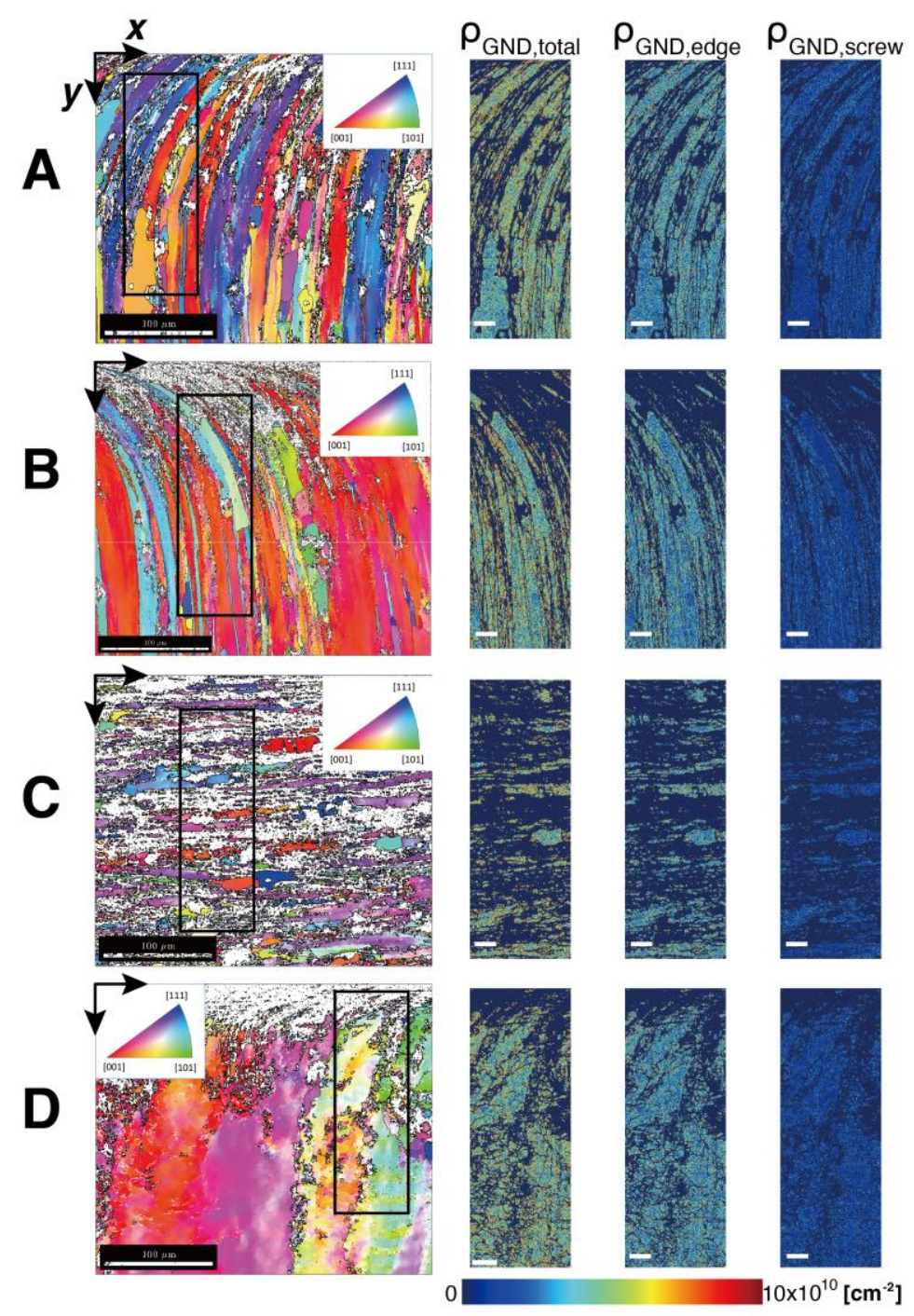

The influence of grain morphology on shear band propensity in 7039-Al alloy with shear zone aligned in the A-, B-, C-, and D-directions is identified by EBSD maps and geometrically-necessary analysis. Rows: $1^{\text {st }}, A ; 2^{\text {nd }}, B ; 3^{\text {rd }}, C ; 4^{\text {th }}, D$. Columns: $1^{\text {st }}$, EBSD image plotted with MTEX with selected example region showing the GND density map (Scale bar: $100 \mu \mathrm{m}$ ); $2^{\text {nd }}$, total GND density map of the selected region ( $\log 10$ scale, unit: $1 / \mathrm{cm}^{2}$ ) with $20 \mu \mathrm{m}$ scale bar; $3^{\text {rd }}$, GND density map of edge type dislocations ( $\log 10$ scale, unit: $1 / \mathrm{cm}^{2}$ ) with $20 \mu \mathrm{m}$ scale bar; $4^{\text {th }}$, GND density map of screw type dislocations ( $\log 10$ scale, unit: $1 / \mathrm{cm}^{2}$ ) with $20 \mu \mathrm{m}$ scale bar. 